Arq. Bras. Med. Vet. Zootec., v.66, n.2, p.462-470, 2014

\title{
Eletroacupuntura para tratamento de hipotensão induzida por isofluorano em cavalos
}

[Electroacupuncture treatment for isoflurane induced hypotension in horses]

\author{
E.C. Franco, R.N. Cassu*, M.S. Diniz, G.M. Mattos, P.C. Scarcelli
}

Faculdade de Ciências Agrárias - UNOESTE - Presidente Prudente, SP

\begin{abstract}
RESUMO
Objetivou-se avaliar a eficiência do tratamento da hipotensão arterial com eletroacupuntura comparativamente à dobutamina em equinos. Foram avaliados seis cavalos adultos, saudáveis, mantidos em anestesia inalatória, com isofluorano, em ventilação mecânica. Após a estabilização da anestesia, foi induzida hipotensão arterial, através do incremento da concentração do isofluorano, iniciando-se um dos tratamentos: DOB: dobutamina $\left(1,5 \mu \mathrm{g} \mathrm{kg}^{-1} \mathrm{~min}^{-1}\right.$, infusão contínua intravenosa); EA: estímulo elétrico no acuponto pericárdio 6 (PC6), bilateralmente; SHAM: estímulo elétrico em ponto falso de acupuntura. Foram mensurados: frequência cardíaca (FC), pressão arterial média (PAM), temperatura retal (T), concentração final expirada de isofluorano $\left(\mathrm{ET}_{\text {iso }}\right.$ ), variáveis hemogasométricas, concentração sérica de aspartato aminotransferase (AST) e creatina fosfoquinase (CK), tempo e qualidade da recuperação pósanestésica. Houve incremento na PAM de 50\%, 36,6\% e 7,5\% nos tratamentos DOB, EA e SHAM, respectivamente. Não houve diferença entre os grupos nas variáveis hemogasométricas, FC, T, ET $\mathrm{Eso}_{\text {iso }} \mathrm{CK}_{\text {, }}$ AST, tempo e qualidade de recuperação pós-anestésica. Conclui-se que o tratamento com dobutamina foi mais efetivo para o tratamento da hipotensão em cavalos sob anestesia inalatória quando comparado ao estímulo elétrico do acuponto PC6 ou ponto falso de acupuntura.
\end{abstract}

Palavras-chave: equino, eletroacupuntura, dobutamina, hipotensão

\begin{abstract}
This study aimed to evaluate the efficacy of electroacupuncture compared to the dobutamine treatment of hypotension in equines. Six adult horses were maintained in isoflurane anesthesia with mechanical ventilation. After anesthesia was established, the isoflurane concentration was raised until hypotension was achieved. After that the animals were treated with a constant rate of $1.5 \mathrm{mg} \mathrm{kg}^{-1} \mathrm{~min}^{-1}$ intravenous dobutamine (DOB), electroacupunture to pericardium $6(P C-6)$ acupoint $(E A)$ and false point treatment $(S H A M)$. Heart rate $(H H)$, median arterial blood pressure $(M A P)$, rectal temperature $(T)$, isoflurane endtidal concentration, arterial blood gases, creatine kinase $(C K)$, aspartate transaminase (AST), recovery time and quality of recovery were investigated. The MAP increased 50\%, 36.5\% and 7.5\% in DOB, EA and SHAM treatments, respectively. HH, T, arterial blood gases, CK, AST, recovery time and quality of recovery did not differ among treatments. It was concluded that the dobutamine treatment was more effective than EA and SHAM treatments for the reversion of isoflurane induced hypotension in horses.
\end{abstract}

Keywords: equine, electroacupuncture, dobutamine, hypotension

\section{INTRODUÇÃO}

A hipotensão arterial é uma complicação comum em equinos sob anestesia geral, que está associada ao aumento de morbidade e

Recebido em 17 de setembro de 2013

Aceito em 20 de março de 2014

*Autor para correspondência (corresponding author)

E-mail: renavarro@uol.com.br mortalidade trans e pós-anestésicas (De Vries et al., 2009). O tratamento convencional consiste no emprego de fármacos simpatomiméticos, como dopamina, dobutamina, noradrenalina e efedrina (Valverde et al., 2006). A dobutamina é um dos fármacos de eleição para o tratamento da 
hipotensão em equinos anestesiados, em razão de atuar predominantemente nos receptores $\beta 1$ adrenérgicos, culminando com efeito inotrópico positivo, que resulta em incremento no retorno venoso, débito cardíaco e pressão arterial (Valverde et al., 2006; De Vries et al., 2009). No entanto, por tratar-se de uma catecolamina sintética, a dobutamina pode induzir a ocorrência de arritmia cardíaca dose-dependente (Borer e Clarke, 2006), além de sua eficiência ser prejudicada em animais endotoxêmicos, em função da redução de sensibilidade dos receptores $\beta$ cardíacos (Trim e Moore, 2007).

Dessa forma, é de grande valia a investigação de outras terapias de suporte que sejam capazes de promover estabilidade hemodinâmica, com custo acessível e com mínimos efeitos adversos para o paciente. Nesse contexto, alguns estudos têm demonstrado resultados favoráveis com o emprego da acupuntura para o tratamento da hipotensão arterial em cães (Syuu et al., 2001; 2003), em ratos (Gao et al., 2009) e no homem (Shidong et al., 2004; Saghaei et al., 2005; Arai et al., 2008).

Todavia, apesar da alta incidência de hipotensão arterial em equinos durante a anestesia geral inalatória e dos resultados positivos relatados em outras espécies com o emprego da acupuntura para o restabelecimento das variáveis hemodinâmicas, não foi encontrada, na literatura consultada, estudos com essa modalidade terapêutica para tratamento de cavalos com hipotensão arterial.

Objetivou-se avaliar e comparar a eficiência da eletroacupuntura com a eficiência da dobutamina para o tratamento da hipotensão arterial em equinos induzida pela anestesia geral inalatória. Paralelamente, objetivou-se avaliar as alterações nas concentrações séricas das enzimas CK e AST, bem como o tempo e a qualidade da recuperação pós-anestésica.

\section{MATERIAL E MÉTODOS}

Após aprovação da Comissão de Ética de Uso de Animais na Experimentação (CEUA) da Instituição de Origem (protocolo n. 232/2009), foram avaliados seis cavalos (1 macho e 5 fêmeas), adultos, com peso médio de $374 \pm 50 \mathrm{~kg}$, saudáveis, selecionados mediante a normalidade dos exames físico e laboratorial (hemograma, função hepática e renal). Os animais foram mantidos em piquete, com acesso à alimentação com coast cross, sal mineral e água ad libitum. Todos os cavalos participaram de todos os tratamentos, com intervalo de 15 dias entre cada avaliação para um mesmo animal.

Após o período de jejum sólido de 12 horas, todos os animais foram submetidos ao mesmo protocolo anestésico. Na sedação foi administrado pela via intravenosa (IV) $0,5 \mathrm{mg} \mathrm{kg}$ ${ }^{1}$ de xilazina (Sedazine 10\%, Fort Dodge Saúde Animal, São Paulo, Brasil). Quinze minutos depois realizou-se a indução anestésica com éter gliceril guaiacol (EGG, Henrifarma Prod. Químicos e Farm. Ltda., São Paulo, Brasil), na dose de $100 \mathrm{mg} \mathrm{kg}^{-1}$ (IV), diluído em solução fisiológica, em concentração de $10 \%$ em associação a $5 \mathrm{mg} \mathrm{kg}^{-1}$ (IV) de tiopental sódico (Tiopentax, Cristália, Itapira, Brasil). Ato contínuo, foi realizada a intubação endotraqueal, com conexão da sonda ao circuito circular valvular semifechado do aparelho de anestesia (Pegasus Plus, Serra, Espírito Santo, Brasil), pelo qual foi fornecido oxigênio a $100 \%\left(10 \mathrm{ml} \mathrm{kg}^{-1}\right.$ $\mathrm{min}^{-1}$ ), com manutenção sob anestesia geral inalatória com isofluorano (Isoforine, Cristália, Itapira, Brasil), cuja concentração final expirada (ETiso) foi ajustada com analisador de gases (Monitor VAMOS plus, Dräger Safety do Brazil, São Paulo, Brasil), sendo mantida em valor aproximado de $1,44 \%$, equivalente a 1 CAM (Steffey et al, 2002).

Os animais foram mantidos em decúbito lateral esquerdo em ventilação mecânica, que foi ajustada com pressão de admissão variando entre 20-25 $\mathrm{cmH}_{2} \mathrm{O}$, frequência respiratória entre 4 a 6 mov $\mathrm{min}^{-1}$, volume corrente entre 15 a $20 \mathrm{ml} \mathrm{kg}^{-1}$, visando manter a concentração final expirada de dióxido de carbono $\left(\mathrm{ETCO}_{2}\right)$ entre $35-45 \mathrm{mmHg}$, mensurada através de capnografia (Monitor VAMOS plus, Dräger Safety do Brasil, São Paulo, Brasil).

Após a estabilização da anestesia, foi realizada a cateterização da artéria facial direita e a adaptação dos eletrodos nos membros torácicos e pélvicos para a realização de eletrocardiografia. Depois de concluída a instrumentação do animal, foi induzida a hipotensão arterial (PAM entre 40 a $45 \mathrm{mmHg}$, mediante o incremento da concentração expirada de isofluorano (Valverde et al., 2006). A partir do estabelecimento da 
hipotensão, os animais foram submetidos a um dos três tratamentos: DOB - administração de dobutamina (Dobutanil, Novafarma, São Paulo, Brasil) $\left(1,5 \mu \mathrm{g} \mathrm{kg}^{-1} \mathrm{~min}^{-1}\right)$ em infusão contínua intravenosa, até a estabilidade da PAM, ou até obtenção máxima de PAM de $100 \mathrm{mmHg}$, momento em que a infusão foi interrompida; EA - aplicação de eletroacupuntura no acuponto PC6, localizado na borda cranial da castanha, na face medial do membro torácico. As agulhas (Suzhou Huanqiu, Acupuncture Medical Applance, CO, Ltda, China) foram introduzidas no referido ponto bilateralmente, com adaptação dos eletrodos no corpo das mesmas, com o aparelho de eletroacupuntura (Sikuro, DS100, Rio de Janeiro, Brasil) desligado. O estímulo de baixa frequência $(3-10 \mathrm{~Hz})$ foi iniciado com corrente zero, elevando-se a mesma, lentamente, mantendo-se o estímulo suficiente para observação de contrações musculares localizadas; SHAM - tratamento similar ao realizado no EA, porém as agulhas foram introduzidas em pontos falsos de acupuntura, localizados próximos aos pontos verdadeiros, porém em locais por onde não percorrem os meridianos principais (Cassu et al., 2008).

Em casos de animais refratários, após 30 minutos de tratamento com estímulo elétrico no PC6 (EA) ou em ponto falso de acupuntura (SHAM), foi administrada infusão de dobutamina, de forma semelhante ao descrito no tratamento DOB.

Foram avaliados: frequência cardíaca (FC) e ritmo cardíaco, por meio de eletrocardiografia (TEB ECG, São Paulo, Brasil); pressão arterial média invasiva (PAM), pela cateterização da artéria facial com leitura em manômetro aneroide (BIC, SP); temperatura retal (T), pela introdução do termômetro digital (BD, MG.) na ampola retal, durante 2 minutos; variáveis hemogasométricas, mediante colheita de sangue da artéria facial, em seringas previamente heparinizadas para avaliação da pressão parcial de dióxido de carbono $\left(\mathrm{PaCO}_{2}\right)$, pressão parcial de oxigênio $\left(\mathrm{PaO}_{2}\right)$, saturação de oxigênio na hemoglobina $\left(\mathrm{SatO}_{2}\right)$, bicarbonato $\left(\mathrm{HCO}_{3}{ }^{-}\right)$ e concentração hidrogeniônica $(\mathrm{pH})$. Para realização de cada exame, o hemogasômetro (Cobasp, modelo 121, Roche-Macromed, São Paulo, Brasil) foi ajustado de acordo com a temperatura retal do animal.

Os parâmetros foram avaliados após a estabilização da anestesia geral e instrumentação do animal (M0), no momento da obtenção da hipotensão arterial (M1) e a cada 10 minutos (M10, M20, M30, M40, M50 e M60), após o início de cada um dos tratamentos, durante 60 minutos. Exceção à temperatura retal e à hemogasometria, que foram mensuradas apenas no M0, M30 e M60.

Foram colhidas amostras de sangue da veia jugular para mensuração da concentração sérica das enzimas AST e CK, pelo método cinético UV, feito em analisador semiautomático (Quick Lab-Drake, São Paulo, Brasil), antes do procedimento (Basal), ao término do procedimento (Pós-anestesia), 4 e 24 horas após o término da anestesia.

Foram aferidos também o tempo médio de anestesia, o tempo médio de recuperação pósanestésica (RPA) (período transcorrido a partir da interrupção da anestesia inalatória até a permanência do animal em posição quadrupedal) e a qualidade da RPA, avaliada mediante escore (Tab. 1).

A estatística foi feita com Análise de Variância (ANOVA), seguindo-se o teste de Tukey para comparar diferenças entre as médias dos diferentes grupos, para variáveis paramétricas. Os escores obtidos da qualidade de RPA foram avaliados por Análise de Variância (ANOVA), seguida pelo teste de Dunn. As análises foram realizadas em microcomputador padrão PC, empregando-se programa Graphpad Instat5, com nível de significância de $5 \%$. 
Tabela 1. Critérios empregados para avaliação da qualidade da recuperação pós-anestésica e número de animais em cada categoria

\begin{tabular}{|c|c|c|c|c|}
\hline \multirow{2}{*}{ Critério } & \multirow{2}{*}{ Escore } & \multicolumn{3}{|c|}{$\mathrm{N}^{\circ}$ de animais } \\
\hline & & $\mathrm{DOB}$ & EA & Sham \\
\hline $\begin{array}{l}\text { Excelente } \\
\text { Sem dificuldade de assumir a posição quadrupedal } \\
\text { Adequada }\end{array}$ & 5 & 2 & 3 & 2 \\
\hline $\begin{array}{l}\text { Ataxia discreta, porém o animal é capaz de assumir a posição quadrupedal } \\
\text { com estabilidade }\end{array}$ & 4 & 2 & 3 & 2 \\
\hline $\begin{array}{l}\text { Tolerável } \\
\text { Ataxia moderada, com } 2 \text { ou } 3 \text { insucessos para assumir a posição } \\
\text { quadrupedal } \\
\text { Insatisfatória }\end{array}$ & 3 & 2 & 0 & 1 \\
\hline $\begin{array}{l}\text { Ataxia grave, delírio e excitação, presença de pedalagem, quedas durante as } \\
\text { tentativas para assumir a posição quadrupedal } \\
\text { Péssima }\end{array}$ & 2 & 0 & 0 & 1 \\
\hline $\begin{array}{l}\text { Impossibilidade de assumir a posição quadrupedal após } 180 \text { minutos do } \\
\text { término da anestesia }\end{array}$ & 1 & 0 & 0 & 0 \\
\hline
\end{tabular}

Fonte: Young e Taylor, 1993.

\section{RESULTADOS}

Os tempos médios de instrumentação $(26 \pm 3$, $19 \pm 9,24 \pm 7$ minutos nos tratamentos DOB, EA e SHAM, respectivamente) e o tempo médio demandado para obtenção da queda da pressão arterial nos valores de $40-45 \mathrm{mmHg}(7 \pm 3,8 \pm 4$, $7 \pm 6$ minutos nos tratamentos DOB, EA e SHAM, respectivamente) não diferiram entre os grupos.

Com relação à $\mathrm{FC}$, ao ritmo cardíaco e às variáveis hemogasométricas $\left(\mathrm{PaCO}_{2}, \mathrm{PaO}_{2}\right.$, $\mathrm{HCO}_{3}^{-}$e $\mathrm{pH}$ ), não foram observadas diferenças entre os grupos nem ao longo do tempo (Tab. 2). Houve incremento da PAM de 50\%, 36,6\% e 7,5\% após 30 minutos de tratamento nos grupos DOB, EA e SHAM, respectivamente. Valores superiores de PAM foram observados no tratamento DOB em relação ao tratamento SHAM aos 10, 20, 30 e 40 minutos, e aos 20 minutos em relação ao tratamento EA. Paralelamente, aos 40 minutos, a PAM no tratamento EA foi significativamente superior em relação ao tratamento SHAM (Tab. 2). Na comparação entre momentos, houve aumento significativo na PAM em relação aos valores registrados no M0 e M1 a partir de 20 e 30 minutos após o início do tratamento com dobutamina e EA, respectivamente. No tratamento SHAM, aos 40 minutos foram observados valores superiores de PAM em relação aos momentos M0, M1, M10 e M20 (Tab. 2).
A temperatura retal reduziu progressivamente em todos os tratamentos sem diferença significativa entre os grupos ou entre os momentos avaliados (Tab. 2).

Os tempos médios de anestesia $(103 \pm 10$, $105 \pm 26,101 \pm 20$ minutos, nos tratamentos DOB, EA e SHAM, respectivamente) e de RPA $(50 \pm 12,51 \pm 26,51 \pm 17$ minutos nos tratamentos DOB, EA e SHAM, respectivamente) não diferiram entre os grupos.

A qualidade da RPA foi semelhante entre os tratamentos, sendo atribuído escore 5 para $33,3 \%$ (2/6) dos animais dos tratamentos DOB e SHAM e $50 \%(3 / 6)$ dos animais do tratamento EA (Tab. 1).

Com relação às enzimas $\mathrm{CK}$ e $\mathrm{AST}$, não foram observadas diferenças entre os grupos. Contudo, na comparação entre momentos, houve aumento significativo na CK às 24 horas de avaliação em relação aos valores basais no tratamento DOB e SHAM. Paralelamente, no tratamento SHAM, valores superiores de CK também foram observados 4 horas após o término da anestesia em relação aos valores basais e aos valores observados ao término da anestesia (Fig. 1A). No tratamento EA, a CK não diferiu ao longo do tempo. A AST aumentou 24 horas após o término da anestesia em todos os tratamentos em relação aos valores basais (Fig. 1B). 


\section{Franco et al.}

Tabela 2. Valores médios e desvio padrão da frequência cardíaca (FC), pressão arterial media (PAM), temperatura retal (T), fração expirada de isofluorano (ETiso), pressão parcial de dióxido de carbônico $\left(\mathrm{PaCO}_{2}\right)$, pressão parcial de oxigênio $\left(\mathrm{PaO}_{2}\right)$, bicarbonato $\left(\mathrm{HCO}_{3}^{-}\right)$e concentração hidrogeniônica $(\mathrm{pH})$ no sangue arterial de cavalos tratados com dobutamina (DOB), eletroacupuntura (EA) e pontos falsos de acupuntura (Sham)

\begin{tabular}{|c|c|c|c|c|c|c|c|c|}
\hline $\begin{array}{l}\text { FC } \\
\text { (bat/min) }\end{array}$ & M0 & M1 & M10 & M20 & M30 & M40 & M50 & M60 \\
\hline DOB & $42 \pm 6$ & $35 \pm 7$ & $39 \pm 9$ & $42 \pm 11$ & $42 \pm 9$ & $44 \pm 10$ & $43 \pm 11$ & $43 \pm 8$ \\
\hline EA & $40 \pm 8$ & $37 \pm 7$ & $40 \pm 7$ & $36 \pm 7$ & $36 \pm 8$ & $38 \pm 7$ & $37 \pm 6$ & $35 \pm 5$ \\
\hline SHAM & $40 \pm 6$ & $41 \pm 7$ & $38 \pm 5$ & $37 \pm 4$ & $38 \pm 4$ & $39 \pm 5$ & $41 \pm 8$ & $43 \pm 8$ \\
\hline \multicolumn{9}{|c|}{ PAM $(\mathrm{mmHg})$} \\
\hline \multicolumn{9}{|c|}{ DOP } \\
\hline EA & $45 \pm 8$ & $42 \pm 4$ & $49 \pm 6$ & $52 \pm 5$ & $62 \pm 8^{\circ}$ & $72 \pm 8 \bullet \circ$ & $80 \pm 15^{\circ}$ & $83 \pm 15^{\circ}$ \\
\hline SHAM & $50 \pm 5$ & $44 \pm 3$ & $46 \pm 4$ & $50 \pm 5$ & $53 \pm 6$ & $65 \pm 2 *$ & $74 \pm 5^{\circ}$ & $82 \pm 7^{*}$ \\
\hline \multicolumn{9}{|l|}{$\mathrm{T}\left({ }^{\circ} \mathrm{C}\right)$} \\
\hline DOB & $37,1 \pm 0$ & & & & $36,3 \pm 1$ & & & $36 \pm 1$ \\
\hline EA & $37,1 \pm 0$ & & & & $36,5 \pm 0$ & & & $36,1 \pm 1$ \\
\hline SHAM & $37,3 \pm 1$ & & & & $36,6 \pm 0$ & & & $36,6 \pm 0$ \\
\hline \multicolumn{9}{|l|}{ ETiso (\%) } \\
\hline DOB & $1,88 \pm 0,7$ & $1,85 \pm 0,3$ & $1,73 \pm 0,2$ & $1,71 \pm 0,3$ & $1,60 \pm 0,2$ & $1,60 \pm 0,1$ & $1,70 \pm 0,3$ & $1,40 \pm 0,2$ \\
\hline EA & $1,70 \pm 0,3$ & $1,85 \pm 0,3$ & $1,50 \pm 0,1$ & $1,40 \pm 0,2$ & $1,40 \pm 0,1$ & $1,50 \pm 0,2$ & $1,30 \pm 0,2$ & $1,30 \pm 0,2$ \\
\hline SHAM & $1,70 \pm 0,4$ & $1,80 \pm 0,4$ & $1,70 \pm 0,4$ & $1,60 \pm 0,3$ & $1,60 \pm 0,3$ & $1,55 \pm 0,5$ & $1,50 \pm 0,5$ & $1,50 \pm 0,5$ \\
\hline \multicolumn{9}{|c|}{$\mathrm{PaCO}_{2}(\mathrm{mmHg})$} \\
\hline DOB & $33 \pm 7$ & & & & $39 \pm 9$ & & & $39 \pm 7$ \\
\hline EA & $33 \pm 4$ & & & & $35 \pm 5$ & & & $41 \pm 4$ \\
\hline SHAM & $43 \pm 9$ & & & & $33 \pm 5$ & & & $40 \pm 7$ \\
\hline \multicolumn{9}{|c|}{$\mathrm{PaO}_{2}(\mathrm{mmHg})$} \\
\hline DOB & $203 \pm 42$ & & & & $255 \pm 31$ & & & $254 \pm 55$ \\
\hline EA & $215 \pm 59$ & & & & $228 \pm 44$ & & & $248 \pm 40$ \\
\hline SHAM & $200 \pm 17$ & & & & $225 \pm 27$ & & & $230 \pm 28$ \\
\hline \multicolumn{9}{|c|}{$\mathrm{HCO}_{3}^{-1}(\mathrm{mEq} / \mathrm{L})$} \\
\hline DOB & $20 \pm 3$ & & & & $22 \pm 2$ & & & $21 \pm 1$ \\
\hline EA & $21 \pm 1$ & & & & $20 \pm 2$ & & & $22 \pm 2$ \\
\hline SHAM & $20 \pm 2$ & & & & $19 \pm 2$ & & & $21 \pm 2$ \\
\hline \multicolumn{9}{|c|}{$\mathrm{pH}$ (unidades) } \\
\hline DOB & $7,37 \pm 0$ & & & & $7,35 \pm 0$ & & & $7,35 \pm 0$ \\
\hline EA & $7,42 \pm 0$ & & & & $7,39 \pm 0$ & & & $7,35 \pm 0$ \\
\hline SHAM & $7,40 \pm 0$ & & & & $7,39 \pm 0$ & & & $7,34 \pm 0$ \\
\hline
\end{tabular}

${ }^{\#} \mathrm{P}<0,05$ : diferença significativa entre os tratamentos DOB e Sham; $* \mathrm{P}<0,05$ : diferença significativa entre os tratamentos DOB e EA, em relação aos momentos $\mathrm{M} 0$ e $\mathrm{M} 1 ; \cdot \mathrm{P}<0,05$ : diferença significativa entre os tratamentos EA e Sham; ${ }^{\circ} \mathrm{P}<0,05$ : aumento significativo em relação à $\mathrm{M} 0$ e $\mathrm{M} 1 ;{ }^{*} \mathrm{P}<0,05$ : aumento significativo em relação à $\mathrm{M} 0$, M1, M10 e M20. 


\section{DISCUSSÃO}

Habitualmente, na Medicina Tradicional Chinesa, o Pericárdio 6 (PC-6) é um dos principais pontos empregados para o tratamento de desequilíbrios relacionados ao sistema cardiovascular. Estudos prévios têm relatado incremento do volume sistólico, pressão arterial e débito cardíaco com o estímulo desse acuponto (Syuu et al., 2001; Syuu et al., 2003; 2004). Syuu et al. (2001) observaram aumento de $15 \%$ nas variáveis hemodinâmicas, após o tratamento com EA no PC6, em cães sob anestesia geral, concordando com os achados do presente estudo, no qual um incremento de $36 \%$ foi observado na PAM, após 30 minutos de tratamento com EA.

O exato mecanismo de ação pelo qual a acupuntura ativa o sistema cardiovascular não está completamente elucidado. Hipoteticamente, a introdução da agulha associada à aplicação de um estímulo elétrico, capaz de promover discretas contrações musculares, poderia estimular o sistema nervoso autônomo simpático, como resultado da ativação de fibras nociceptivas, devido a um possível desconforto ao paciente (Syuu et al., 2003). Todavia, os animais do atual estudo foram mantidos em plano anestésico capaz de inibir a resposta nociceptiva induzida por um estímulo de baixa intensidade, como o desencadeado pela EA, sugerindo que a ativação do sistema cardiovascular não teve uma relação direta ao estímulo nociceptivo. Ademais, se a introdução da agulha acrescida do estímulo elétrico tivesse gerado desconforto nos animais, era esperado que o grupo tratado com pontos falsos apresentasse incremento na PAM equivalente ao grupo tratado com EA. Contudo, nos animais do tratamento SHAM, apenas um discreto aumento $(7,5 \%)$ foi observado nessa variável, demonstrando a necessidade do estímulo em ponto verdadeiro de acupuntura para obtenção de resposta mais efetiva. Em estudo similar, Syuu et al. (2003) observaram aumento nas variáveis hemodinâmicas de cães tratados com pontos falsos de acupuntura, porém a resposta foi de menor magnitude em relação ao tratamento com EA no PC6. Resultados semelhantes foram descritos por Arai et al. (2008), que observaram aumento mais expressivo da pressão arterial de mulheres tratadas com estímulo elétrico em pontos de acupuntura quando comparadas ao tratamento em pontos falsos, sugerindo que a aplicação do estímulo elétrico contribui para o incremento da pressão arterial, porém o resultado é mais efetivo quando o estímulo é aplicado no ponto de acupuntura.

O acuponto PC6 localiza-se próximo ao nervo mediano, cujas raízes originam-se dos ramos ventrais e dorsais, compreendidos entre a quinta vértebra cervical até a primeira vértebra torácica, com projeções para o centro vasomotor e cardíaco, localizados no bulbo (Li et al., 2006). O estímulo aplicado no acuponto PC6 é capaz de ativar centros espinhais e supraespinhais, devido à projeção segmentar do trajeto neural. Dessa forma, quando os neurônios da medula espinhal são ativados por impulsos aferentes da EA, os mesmos são capazes de estimular os neurônios adjacentes, favorecendo a ativação do centro vasomotor, que resulta em estímulo simpático, com aumento da pressão arterial (Syuu et al., 2003).

Resultados mais eficientes foram observados no grupo DOB, cujo aumento da PAM foi observado a partir dos 20 minutos de tratamento, concordando com resultados prévios que demonstraram estabilidade desse parâmetro após $17 \pm 4$ minutos de infusão de dobutamina em cavalos anestesiados pelo isofluorano (De Vries et al., 2009). Era esperado que o grupo tratado com dobutamina restabelecesse mais rapidamente a pressão arterial, devido ao curto período de latência desse fármaco, que pode variar entre 2 a 20 minutos, em dependência da dose empregada (Benedikter e Mey, 1981). Já nos animais tratados com EA, o incremento da PAM em relação ao basal foi mais tardio, corroborando outros autores que demonstraram que o tempo médio para a obtenção de efeito estimulante cardiovascular com EA foi cerca de 30 minutos após o início do tratamento (Syuu et al., 2003; Gao et al., 2009). No entanto, apesar do incremento na PAM, apenas dois dos seis animais $(33,3 \%)$ do grupo EA atingiram valores iguais ou superiores a $70 \mathrm{mmHg}$, de modo que a suplementação com dobutamina foi necessária em quatro animais. No tratamento SHAM, o resultado foi menos promissor, sendo instituída a infusão de dobutamina em $100 \%$ dos animais, pois em nenhum deles o tratamento foi efetivo.

Apesar de a resposta do tratamento com EA ter sido inferior quando comparada à da dobutamina, estatisticamente diferença na PAM 
só foi observada aos 20 minutos de avaliação. Contudo, do ponto de vista clínico, o tratamento com dobutamina mostrou-se mais efetivo, visto que todos os animais responderam ao tratamento, ao passo que, no grupo EA, a resposta foi parcial. Todavia, pesquisas que avaliaram o tratamento com acupuntura em pacientes anestesiados submetidos a diferentes cirurgias obtiveram respostas promissoras, sugerindo que a ativação simpática promovida pelo estímulo nociceptivo cirúrgico possa contribuir, favorecendo a ativação cardiovascular (Syuu et al., 2001; Arai et al., 2008). Dessa forma, apesar de o atual estudo não ter avaliado o uso da acupuntura em situações clínicas, é provável que, na presença do estímulo cirúrgico, o efeito pudesse ser mais efetivo, visto que, mesmo em condições de hipotensão severa $(42 \pm 8 \mathrm{mmHg})$, a EA conseguiu elevar a PAM para $62 \pm 8 \mathrm{mmHg}$ após 30 minutos de tratamento, sem o uso de nenhum fármaco simpatomimético, mesmo sob depressão central, promovida pela anestesia geral. Tanto o isofluorano como o tiopental deprimem a resposta barorreflexa (Skovsted et al., 1970; Seagard et al., 1983). O isofluorano, quando empregado em até 1 CAM em cães normotensos, manteve intactos os barorreceptores, sendo comprovada a depressão barorreflexa a partir de 2 CAM. Porém, em situações de hipotensão, os barorreceptores foram inibidos a partir de 1 CAM de isofluorano, não sendo observada a taquicardia reflexa (Seagard et al., 1983). Esses achados são consistentes com os resultados do presente estudo, sendo observada estabilidade da FC, sem a identificação de taquicardia em nenhum dos tratamentos, mesmo nos momentos iniciais (basal e M1), em que a PAM estava bem abaixo dos valores fisiológicos para a espécie, sugerindo que a resposta barorreflexa foi inibida pela anestesia, apesar de não ter sido atingida 2 CAM de isofluorano, mesmo para a indução da hipotensão. Alterações de ritmo cardíaco também não foram observadas. Contudo, um animal do grupo DOB desenvolveu taquicardia a partir dos 20 minutos após o início do tratamento, provavelmente devido ao estímulo adrenérgico proporcionado por esse fármaco, visto que nessa ocasião a pressão desse animal já se encontrava estável. Porém, após a interrupção da dobutamina, foi observada estabilidade da FC, não representando relevância clínica ao animal.

Com relação aos parâmetros respiratórios e hemogasométricos, foi observada estabilidade em todos os animais, em função do emprego da ventilação mecânica e do posicionamento dos animais em decúbito lateral esquerdo, que favorecem a redução da ocorrência de atelectasia alveolar e de shunt pulmonar (Braun et al., 2009). Em todos os tratamentos, foi observada discreta redução do $\mathrm{pH}$, em função do aumento da $\mathrm{PaCO}_{2}$, porém essas alterações não apresentaram relevância clínica, visto que os valores observados mantiveram-se próximos dos limites fisiológicos para a espécie equina.

A redução progressiva da temperatura retal ao longo do tempo foi consequência da depressão hipotalâmica e da vasodilatação provocadas pela anestesia geral inalatória (Steffey e Mama, 2007); no entanto, hipotermia não foi evidenciada em nenhum dos animais avaliados, de modo que essa alteração não teve relevância clínica.

Resultados satisfatórios foram relatados com o emprego de fármacos simpatomiméticos, como dobutamina, noradrenalina e dopamina, para o tratamento da hipotensão arterial em cavalos, de modo a minimizar a incidência da miopatia pósanestésica (Valverde et al., 2006; Borer e Clarke, 2006; De Vries et al., 2009), cuja lesão muscular é sinalizada pelo aumento das enzimas AST e CK (Voulgaris e Hofmeister, 2009). No atual estudo, apesar do incremento das referidas enzimas, nenhum animal apresentou sinais clínicos compatíveis com o quadro de lesão muscular, corroborando estudos prévios que relataram mínima incidência de miopatia em animais sob anestesia geral com duração média de até 100 minutos (Young e Taylor, 1993; Duke et al., 2006).

O tempo de RPA foi semelhante ao relatado por outros autores (Valverde et al., 2006, De Vries et al., 2009). Ademais, os escores de RPA variaram entre adequado a excelente para a maioria dos animais, sugerindo que os diferentes tratamentos, bem como a hipotensão de curta duração, não prejudicaram a qualidade da recuperação pósanestésica.

\section{CONCLUSÕES}

O tratamento com dobutamina foi mais efetivo para a reversão da hipotensão arterial em cavalos anestesiados com isofluorano quando comparado à aplicação de estímulo elétrico no acuponto PC6 
e em ponto falso de acupuntura. Contudo, o tratamento com EA promoveu elevação mais pronunciada da PAM em relação ao tratamento Sham. Apesar do incremento nas enzimas CK e AST, a qualidade da recuperação pós-anestésica foi adequada em todos os tratamentos.

\section{REFERÊNCIAS}

ARAI, Y.C.P.; KATO, N.; MATSURA, M. et al. Transcutaneous electrical nerve stimulation at the PC-5 and PC-6 acupoints reduced the severity of hypotension after spinal anaesthesia in patients undergoing caesarean section. $\mathrm{Br} . \mathrm{J}$. Anaesth., v.100, p.78-81, 2008.

BENEDIKTER, L.; MEY, T. Onset and magnitude of cardiovascular response to dobutamine and AR-L $115 \mathrm{BS}$, a new positive inotropic agent with additional vasodilating activity, in normal subjects. Arzneimittelforschung, v.31, p.239-242, 1981.

BORER, K.E.; CLARKE, K.W. The effect of hyoscine on dobutamine requirement in spontaneously breathing horses anaesthetized with halothane. Vet. Anaesth. Analg., v.33, p.149-157, 2006.

BRAUN, C.; CYNTHIA, M.; RANDY, B. Effects of changing body position on oxygenation and arterial blood pressures in foals anesthetized with guaifenesin, ketamine, and xylazine. Vet. Anaesth. Analg., v.36, p.18-24, 2009.

CASSU, R.N.; LUNA, S.P.L.; CLARKE, R.M.O. et al. Electroacupuncture analgesia in dogs: is there a difference between uni- and bilateral stimulation? Vet. Anaesth. Analg., v.35, p.52-61, 2008.

DE VRIES, A.; BREARLEY, J.C.; TAYLOR, P.M. Effects of dobutamine on cardiac index and arterial blood pressure in isofluraneanaesthetized horses under clinical conditions. $J$. Vet. Pharmacol. Ther., v.32, p.353-358, 2009.

DUKE, T.; FILZEK, U.; READ, M.R. et al. Clinical observations surrounding an increased incidence of postanesthetic myopathy in halothane-anesthetized horses. Vet. Anaesth. Analg., v.33, p.122-127, 2006.

GAO, Y.H.; CHEN, S.P.; WANG, J.Y. et al. Effects of electroacupuncture of different acupoints groups on blood pressure and heard rate variability in rats. Acup. Res., v.34, p.21-26, 2009.

LI, P.; TJEN-A-LOOI, S.C.; LONGHURST, J.C. Excitatory projections from arcuate nucleus to ventrolateral periaqueductal gray in electroacupuncture inhibition of cardiovascular reflexes. Am. J. Physiol. Heart Circ. Physiol., v.290, p.2535-2542, 2006.

SAGHAEI, M.; AHMADI, A.; REZVANI, M. Clinical trial of nitroglycerin-induced controlled hypotension with or without acupoint electrical stimulation in microscopic middle ear surgery under general anesthesia with halothane. Acta Anaesthesiol. Taiwan., v.43, p.135-139, 2005.

SEAGARD, J.L.; ELEGBE, E.; HOPP, F.A. et al. Effects of isoflurane on the baroreceptor reflex. Anesthesiology, v.59, p.11-20, 1983.

SHIDONG, Y.; YINGJIE, C.; JUN, Z. Treatment of primary hypotension by eletroacupuncture at neiguan and gongsun- a report of 100 cases. $J$. Trad. Chin. Med. v.24, p.193, 2004.

SKOVSTED, P.; PRICE, M.L.; PRICE, H.L. The effects of short-acting barbiturates on arterial pressure, preganglionic sympathetic activity and barostatic reflexes. Anesthesiology, v.33, p.10-18, 1970.

STEFFEY, E.P.; MAMA, K.R. Inhalation anesthetics. In: Tranquilli, W.J.; Thurmon, J.C.; Grimm, K.A. Lumb \& Jones' Veterinary Anesthesia and Analgesia. 4.ed. Oxford: Blackwell Publishing, 2007. Cap.13, p.355-394.

STEFFEY, E.P.; PASCOE, T.J.; WOLINER, M.J. Detomidine reduces isoflurano anesthetic requeriment (MAC) in horses. . Vet. Anaesth. Analg., v.29, p.223-227, 2002.

SYUU, Y.; MATSUBARA, H.; KIYOOKA, T. et al. Cardiovascular beneficial effects of electroacupuncture at Neiguan (PC-6) acupoint in anesthetized open-chest dog. Jpn J. Physiol, v.51, p.231-238, 2001.

SYUU, Y.; MATSUBARA, H.; HOSOGI, S. et al. Pressor effect of electroacupuncture on hemorrhagic hypotension. Am. J. Physiol. Regul. Integr. Comp. Physiol., v.285, p.1446-1452, 2003.

TRIM, C.M.; MOORE, J.M. Horses with colic. In: TRANQUILLI，W.J.; THURMON，J.C.; GRIMM, K.A. Lumb \& Jones' Veterinary 
Anesthesia and Analgesia. Oxford: Blackwell Publishing, 2007, Cap. 51. p.1019-1026.

VALVERDE, A.; GIGUÈRE, S.; SANCHEZ, L.C. et al. Effects of dobutamine, norepinephrine and vasopressin on cardiovascular function in anesthetized neonatal foals with induced hypotension. Am. J. Vet. Res., v.67, p.1730-1737, 2006.
VOULGARIS, D.A.; HOFMEISTER, E.H. Multivariate analysis of factors associated with post-anesthetic times to standing in isofluraneanesthetized horses: 381 cases. Vet. Anaesth. Analg., v.36, p.414-420, 2009.

YOUNG, S.S.; TAYLOR, P.M. Factors influencing the outcome of equine anaesthesia: a review of 1,314 cases. Equine Vet. J., v.25, p.147-151, 1993. 